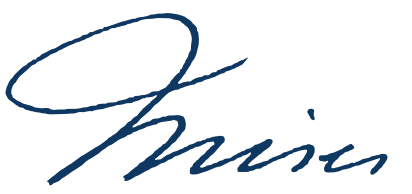

\title{
La Privatización de la Amazonia
}

\author{
Marcos Cantaluppi Plá ${ }^{1}$ (10000-0002-9514-0529
}

Universidad Rey Juan Carlos, Madrid, Espanha

Resumen: Este artículo explora la privatización de la Amazonía. A partir de un ámbito teórico basado en la tragedia de los comunes, el cálculo económico y la eficiencia dinámica, se desarrolla un análisis de las consecuencias de la privatización, tratando de analizar el tema ambiental y cómo se verían afectados los bosques y la contaminación. Se abordan problemas económicos como los costes de contaminación, la visión a corto plazo y la escasez de recursos. Alternativas sostenibles para la explotación forestal están expuestas, y cómo la privatización es un motor para llevar a cabo tales medidas. La privatización de los bosques se ve contrastada con la actual política ambiental, que enfoca en la demarcación de las tierras indígenas y sus consecuencias. Desde un punto de vista económico y ético, que defiende un marco legal de fuerte protección de los derechos de propiedad, se demuestra que la privatización es la mejor solución para la resolución de conflictos y el mantenimiento de la selva amazónica.

Palabras-clave: Amazonia, privatización, medio ambiente, propiedad privada, Economía austriaca.

\footnotetext{
${ }^{\mathrm{I}}$ Mestrando com Qualificação em Escola Austríaca de Economia na Universidade Rey Juan Carlos Madri, Madri, ES.

E-mail: cantaluppi.marcos@gmail.com
} 


\title{
The Privatization of the Amazon
}

\begin{abstract}
This article explores the privatization of the Amazon. Based on a theoretical framework founded upon the tragedy of the commons, economic calculation, and dynamic efficiency, an analysis of the consequences of privatization is developed, trying to assess the environmental issue and how forests and pollution would be affected. Economic problems, such as the costs of pollution, short-term goals, and scarcity of resources are addressed. Sustainable alternatives for logging, and how privatization is an engine to carry out such measures, are exposed. The privatization of the forest is contrasted against the current environmental policy, focusing on the demarcation of indigenous lands and its consequences. From an economic and ethical point of view, defending a legal framework of strong protection of property rights, it is demonstrated that privatization is the best solution for conflict resolution and the maintenance of the Amazon rainforest.
\end{abstract}

Keywords: Amazon, privatization, environment, private property, Austrian economics.

\section{A privatização da Amazônia}

Resumo: Este artigo explora a privatização da Amazônia. Com base em um marco teórico, baseado na tragédia dos comuns, no cálculo econômico e na eficiência dinâmica, é desenvolvida uma análise das consequências da privatização, analisando a questão ambiental e como as florestas e a poluição seriam afetadas. Problemas econômicos, como custos de poluição, visão de curto prazo e escassez de recursos são abordados. São expostas alternativas sustentáveis para a exploração da floresta e discute-se como a privatização é um motor, para levar a cabo tais projetos. A privatização da floresta é contrastada com a atual política ambiental, com foco na demarcação de terras indígenas e suas consequências. Desde um ponto de vista econômico e ético, defendendo uma estrutura legal de forte proteção aos direitos de propriedade, é demonstrado que a privatização é a melhor solução para a resolução de conflitos e manutenção da floresta amazônica.

Palavras-chave: Amazônia, privatização, meio ambiente, propriedade privada, economia austríaca. 


\section{Introducción}

En este artículo, pretendemos analizar la privatización de la Amazonia. Veremos las consecuencias de esta privatización y las contrastaremos con las políticas actuales de preservación de la selva amazónica.

Primero desarrollaremos el marco teórico, pasando por conceptos como la "tragedia de los comunes", el cálculo económico y la eficiencia dinámica, para que podamos entender los problemas que existen en la política actual para la selva amazónica y cuáles son las posibles soluciones. A partir de los conceptos teóricos, estudiaremos los problemas relacionados a una gestión privada de los bosques, teniendo en cuenta sus supuestas ventajas y desventajas. Al final de nuestro trabajo, veremos las peculiaridades de la selva amazónica e intentaremos aplicar los conceptos trabajados aquí.

Este trabajo está motivado por las demandas sociales actuales para combatir el cambio climático. A pesar de que este ensayo está centrado en el tema económico, no siendo aquí el lugar para un análisis climatológico, las medidas actuales planeadas para combatir el calentamiento global siempre demandan una mayor injerencia del Estado en los mercados, exigiendo más regulaciones. Creo que existe una general falta de conocimiento sobre cómo funciona el mercado, siendo importante presentar los problemas causados por estas políticas intervencionistas, para que entonces, logremos encontrar el medio más idóneo de resolver los problemas sociales.

Con relación a esto, la Amazonía, por ser la mayor selva tropical del mundo, es a menudo tema de discusión en la mayoría de las políticas de protección ambiental. Con una amplia variedad de políticas estatales de organismos nacionales y supranacionales y conflictos constantes, se hace necesario comprender el impacto de estas políticas y cómo contribuyen a una solución satisfactoria de los problemas. Con este objetivo, el ensayo intentará mostrar las ventajas de un modelo basado en los derechos de propiedad privada de la selva amazónica.

\section{1. Ámbito Teórico}

\subsection{Tragedia de los Comunes}

El primer gran problema que enfrenta la Amazonía está relacionado a las externalidades negativas derivadas de un bien público. Es decir, es un problema de la "tragedia de los comunes", donde los costes de la contaminación no se internalizan en los precios de los bienes de consumo.

La tragedia de los bienes comunes se da de la siguiente manera: imaginando un pasto abierto a todos. En este escenario, cada ganadero aumentará la cantidad de ganado hasta una situación de hacinamiento, donde las ganancias de una nueva cabeza de ganado generan un coste de igual valor. En este punto, el agricultor podrá detener la expansión del ganado o continuar. El problema surge porque, al aumentar una cabeza de ganado, el agricultor recibirá las ganancias totales de esa adición, mientras que los costes se compartirán entre todos los 
demás ganaderos. Esto genera un incentivo para seguir creciendo su rebaño. Sin embargo, todos los demás ganaderos llegan a la misma conclusión, agotando el recurso (HARDIN, 1968).

Las tierras carentes de dueño efectivo (es indiferente que se consideren propiedad pública desde un punto de vista meramente legal) las utiliza la gente sin preocuparse del daño que puedan sufrir. Cada quien busca lucrar al máximo, por cualquier medio, de sus rentas - madera y caza de los bosques, riqueza piscícola de las aguas, minerales del subsuelodesentendiéndose de los efectos que puedan producirse. La erosión de la tierra, el agotamiento de las riquezas naturales y demás quebrantos futuros son costes externos que los actores no tienen en cuenta en sus cálculos. (MISES, 2011, p. 775)

Los bienes públicos se encuentran en esta condición, no permitiendo la internalización de los costes de uso, generando un problema de externalidades negativas, en el que los costes se socializan entre todos, alentando el agotamiento de los recursos por parte de los usuarios.

Esta situación deja de ocurrir cuando dicho bien tiene un dueño y los derechos sobre su propiedad están bien definidos y protegidos. De esta manera, el propietario será responsable de asumir todos los costes involucrados en la explotación de su recurso, que cesará cuando el coste marginal sea igual al ingreso marginal esperado. Los derechos de propiedad no sólo resuelven la tragedia de los comunes, sino que también los conflictos que pueden involucrar el uso de un bien. Como lo expresó el autor Hans Herman-Hoppe:

To develop the concept of property, it is necessary for goods to be scarce, so that conflicts over the use of these goods can possibly arise. It is the function of property rights to avoid such possible clashes over the use of scarce resources by assigning rights of exclusive ownership. Property is thus a normative concept: a concept designed to make a conflict-free interaction possible by stipulating mutually binding rules of conduct (norms) regarding scarce resources (HOPPE, 1989, p. 8).

En contraste con la posición de Garrett Hardin, Elinor Ostrom nos dice que hay formas de administrar los bienes comunes que no necesariamente conducirían al problema de las externalidades negativas, una vez que un grupo de personas podría organizarse para la gestión colectiva de un bien. De esta manera, los RUC ${ }^{1}$ (recursos de uso común) serían una forma de uso colectivo de un recurso, como se daría en una comunidad de pescadores con un área de pesca. Sin embargo, para que sea posible el surgimiento y mantenimiento de un RUC, implica necesariamente la exclusión del uso del recurso, diferenciándose de un bien público. Los miembros de una comunidad usarán el recurso que es común dentro de él, quedando excluida cualquier persona que esté fuera de la comunidad para evitar el uso excesivo del recurso. Dentro de las comunidades, surgirán reglas y formas de monitoreo que limitarán el uso del recurso, obstaculizarán en mayor medida la acción de los free-riders y generarán incentivos para la cooperación. Las reglas elegidas dependerán de las características específicas de cada caso, en el cual, a través de un proceso evolutivo de prueba y error, prevalecerán las más eficientes.

\footnotetext{
${ }^{1}$ Elinor Ostrom define a los RUC como "un sistema de recursos naturales o hechos por el hombre que es lo suficientemente grande como para volver costoso (pero no imposible) excluir a destinatarios potenciales de los beneficios de su uso". (OSTROM, 2000, p. 66)
} 
Además, para que dicha gestión sea posible, es necesario que las organizaciones sean locales, descentralizadas y pequeñas. De esta manera, evitase que los miembros de una organización actúen de forma independiente, como en el caso del pasto de Hardin. Dicho esquema funciona de manera similar a la gestión de áreas comunes en un edificio, no dejando de ser un sistema de libre mercado, donde la propiedad privada es colectiva (OSTROM, 2000, p. 90).

Por consiguiente, es en realidad el Estado quien crea la Tragedia de los Comunes. Dado que el Estado, como dice Ostrom, crea el "dilema del prisionero" en las situaciones que se exponen, donde los miembros de la cárcel no cooperarán, una vez que se encuentran separados y no pueden comunicarse. No habiendo, por lo tanto, incentivos y formas de establecer un sistema de reglas. "En esta situación actuar de manera independiente es resultado de la coerción, no de su ausencia" (OSTROM, 2000, p. 78). Es decir, el hecho de que no cooperen y establezcan un sistema de reglas que permita el uso común de los recursos y el monitoreo para garantizar si los miembros lo cumplen, como en el caso de las áreas comunes de un edificio, se debe precisamente a la acción coercitiva del Estado. Es evidente, entonces, que el sistema de RUC expuesto por Ostrom es totalmente compatible con el libre mercado.

\subsection{Cálculo Económico}

El segundo gran problema de la Amazonía como bien público es el cálculo económico. En su artículo sobre la imposibilidad del socialismo (1920), Mises explica que en un sistema socialista no hay propiedad privada de los factores de producción, ya que están bajo la propiedad del Estado. Por esa razón, no hay intercambio de factores de producción, es decir, no hay mercado de factores de producción. En consecuencia, no se generan precios de mercado para los factores de producción, no siendo posible hacer una asignación racional de los recursos. En una economía compleja, donde existe una gran variedad de posibilidades de producción para los bienes y servicios de consumo y una variedad mucho más amplia de factores de producción, es imposible valorar la utilidad de cada uno los factores de producción de forma directa. Solamente a través de la institución del dinero, uno de responsables por generar precios de mercado, es posible establecer objetivamente la valoración de los factores de producción con relación a su contribución en los procesos de producción, permitiendo el cálculo del coste y beneficio y, por lo tanto, determinar qué factores de producción son los más adecuados para ser empleado (MISES, 1920).

Dado que el objetivo de toda producción es producir bienes de consumo, los factores de producción se valoran de acuerdo con el precio de los bienes de consumo que producen, que a su vez está subjetivamente determinado por los consumidores. Los empresarios, al estimar cuáles serán las preferencias futuras de los consumidores, asignan los factores de producción $y$, si aciertan, se producen beneficios empresariales. El beneficio significa que el empresario ha asignado correctamente los recursos, donde los consumidores demandan más urgentemente. De esta forma, se coordina un desajuste social.

El problema del cálculo económico ocurre directamente en los bienes públicos, ya que su precio de consumo es cero o el Estado lo define arbitrariamente. Por lo tanto, los precios de mercado no se generan (o se generan de manera distorsionante) para los factores de 
producción que producen bienes públicos. El administrador del bien público desconoce el coste de oportunidad de los recursos. Luego, la asignación de recursos para administrar ese bien es errónea, y mientras mayor es la intervención estatal, es más ineficiente. Por consiguiente, el Estado no tiene la herramienta necesaria ni los incentivos, reflejados en pérdidas y beneficios, para administrar mejor el bien. A continuación, veremos cómo se refleja esto en el caso de la Amazonía.

Para empeorar las cosas, la gestión centralizada del bien hace que ocurra un problema de información. Según autores como Friedrich A. von Hayek y Jesús Huerta de Soto, teóricamente no es posible que un organismo de planificación central pueda obtener toda la información necesaria para llevar a cabo el cálculo económico y cumplir sus mandatos. En primer lugar, debido al volumen, donde el órgano director no puede asimilar el enorme volumen de información que se genera constantemente. En segundo lugar, porque la información que necesita el órgano no es transferible, debido a su naturaleza práctica, subjetiva, tácita y no articulable. Tercero, porque no es posible transmitir información que aún no se ha descubierto o creado. Cuarto, porque la coerción estatal en sí misma bloquea el proceso empresarial de crear y descubrir información (HUERTA DE SOTO, 2005, p. 101-102). Por lo tanto, cuando el estado establece lo que tiene que hacer y cómo debe hacerlo, la transmisión del conocimiento se fosiliza. De esa manera, el gestor del bien público no tiene como saber la forma más eficiente de llevar a cabo sus proyectos, una vez que dicha información no está reflejada en los precios de mercado de dicho bien. El gestor no tiene otro remedio que el mal uso del bien, su mala conservación y el desperdicio de recursos.

\subsection{Eficiencia Dinámica}

El tercer problema que me gustaría abordar en este artículo se refiere a la eficiencia dinámica, definida por Huerta de Soto como "la capacidad para impulsar, por un lado, la creatividad empresarial y, por otro lado, la coordinación, es decir, la capacidad empresarial para buscar, descubrir y superar los diferentes desajustes sociales." (HUERTA DE SOTO, 2004).

Como expuesto por Huerta de Soto, es importante analizar el problema desde esta perspectiva, puesto que la forma en que a menudo es tratado, desde el punto de vista económico y jurídico, se refiere a una visión del mundo estática, donde el único problema económico sería evitar el despilfarro, sin considerar el ser humano como un actor creador. En la visión estática de la eficiencia, el problema se reduce a la determinación del punto óptimo de la curva paretiana, es decir, entre varias posibilidades de redistribución óptima, se elegiría la más deseable. Ya no sería un problema económico, sino de juicios de valor (HUERTA DE SOTO, 2004).

Esta perspectiva estática sobre la eficiencia proviene de pensar que la información necesaria para llevar a cabo un proyecto está dada. Por lo tanto, la forma más eficiente de administrar un bien sería evitar el despilfarro. A medida que analizamos el problema desde un punto de vista dinámico de la eficiencia, se hace evidente que los humanos, al poder aprovecharse de los resultados de su creatividad empresarial, descubrirán nuevas formas de abordar los desajustes para lograr un beneficio empresarial genuino. Por consiguiente, entendemos que un sistema basado en la eficiencia dinámica, con el fin de fomentar la función empresarial, 
es uno que respeta y protege la propiedad privada, de manera que la creación de nuevas alternativas mueve constantemente la curva de posibilidades máximas de producción hacia la derecha. Este sistema, no solo es el más eficiente, sino también el más justo, una vez que, al crear, el actor aprovecha de algo que antes no existía, sin dañar a otros.

Como expuesto por Israel Kirzner, los empresarios están en un constante estado de alerta para descubrir nuevos desajustes sociales, actuando en consecuencia para obtener beneficio. De hecho, el desajuste en el beneficio en el mercado se da justo cuando un empresario es capaz de percibir un desajuste antes que otro. De ese modo, la función empresarial es por naturaleza coordinadora, una vez que corrige un desajuste previo, haciendo que los agentes económicos involucrados aprendan, de manera voluntaria, a coordinar su comportamiento en función de los demás (KIRZNER, 1973, p. 86).

De esa manera, Huerta de Soto argumenta sobre la importancia del libre ejercicio de la función empresarial para generación de información en el mercado:

El ser humano tiende a descubrir la información que le interesa, por lo que, si existe libertad en cuanto a la consecución de fines e intereses, estos mismos actuarán como incentivo, y harán posible que aquel que ejerce la función empresarial motivada por dicho incentivo perciba y descubra continuamente la información práctica relevante para la consecución de los fines propuestos. $\mathrm{Y}$ al revés, si por cualquier razón se acota o se cierra el campo para el ejercicio de la empresarialidad en determinada área de la vida social (mediante restricciones coactivas de tipo legal o institucional), entonces los seres humanos ni siquiera se plantearán la posibilidad de lograr o alcanzar fines en esas áreas prohibidas o limitadas, por lo que, al no ser posible el fin, éste no actuará como incentivo, y como consecuencia de ello tampoco se percibirá ni descubrirá la información práctica relevante para la consecución del mismo. Es más, ni siquiera las personas afectadas serán conscientes en estas circunstancias del gran valor y elevado número de fines que dejan de poder ser logrados como consecuencia de esa situación de restricción institucional. (HUERTA DE SOTO, 2005, p. 74-75)

El libre ejercicio de la función empresarial resuelve el problema de la gestión y agotamiento de los recursos, ya cuando se genera la información de que un recurso es valorado por la sociedad, los agentes involucrados pasan a preservar dicho recurso. Además, cuanto menor es la cantidad de un recurso dado, mayor es su precio de mercado y, por lo tanto, mayores son las oportunidades de beneficio. En un entorno de libertad de mercado, el empresario tendrá un gran incentivo para obtener dicho recurso y preservarlo. Como lo expuesto por autores como Terry Anderson y Donald Leal, la creación de safaris privados en el sur de África fue responsable por resolver el conflicto entre las comunidades locales y la vida silvestre. Como resultado, ha mejorado el nivel de vida de las comunidades y ha contribuido a la preservación de la vida silvestre y de las especies en peligro de extinción. A partir de la acción empresarial, las comunidades pasaran a preservar algo que antes era un problema para ellas, una vez que se creó la información de que era lucrativo la preservación de dicho recurso. Además, buscando la preservación de animales de valor comercial, es decir, los grandes mamíferos, se preservó todo el ecosistema de la región (ANDERSON; LEAL, 2001, p. 153-155).

A continuación, veremos cómo aplicar el criterio dinámico de eficiencia con respecto a la Amazonía. Sin embargo, es importante comprender que a través de la eficiencia dinámica el 
problema ya no es simplemente técnico, habiendo la posibilidad de crearse nuevas alternativas que no se habían desarrollado antes. Esto solo puede ocurrir dentro de un marco jurídico de defensa de la propiedad privada, donde la información que el emprendedor necesita para llevar a cabo sus proyectos no está bloqueada y se puede haber el pleno ejercicio de la función empresarial.

\section{Problemas Relacionados con la Privatización}

\subsection{Costes de Polución}

Uno de los principales problemas en torno a la privatización del medio ambiente, es la afirmación de que el mercado no consigue internalizar los costes de contaminación en los productos. Por lo tanto, la intervención estatal es necesaria para corregir esta falla del mercado, ya sea a través de los impuestos recaudados para una actividad en particular o mediante la propiedad estatal del bien. La intervención sería el único camino de evitar que las empresas sobreexploten los recursos, causando gran polución, contaminación y desastres ambientales, perjudicando a todos y contribuyendo al calentamiento global. Veremos si este análisis es válido.

Primero, como ya hemos visto en el caso de la tragedia de los bienes comunes, el bien público es responsable por generar un problema de externalidades negativas. Por lo tanto, la supuesta solución en la que el bien debe de estar en posesión del Estado, no es válida.

En segundo lugar, la afirmación de que el mercado no internaliza los costes de la contaminación no se debe a una falla del mercado. Todo lo contrario. Lo que sucede, es que en ausencia de una fuerte defensa de los derechos de propiedad, de manera que vigile y castigue las acciones contra la propiedad privada, un pilar que permite la existencia de un mercado, los costes de la polución no pueden ser internalizados. Por lo tanto, no es una falla del mercado, sino una falta de este. Imaginemos la siguiente situación: un terrateniente decide quemar parte de su propiedad para hacerla pastura. En esta suposición, con una fuerte defensa de la propiedad privada, él tendría algunos problemas a considerar. 1) ¿La contaminación por la quema afectará otras propiedades?; 2) Si es así, ¿qué compensación tendré que pagar por los daños a la propiedad de terceros?; 3) ¿Las ganancias del pastoreo futuro menos el coste de la compensación serán lo suficientemente altas como para ser más ventajoso que otro uso económico de la tierra? Lo que ocurre, es que los costes de la contaminación se harían reflejados en el precio del producto final de este tipo de actividad económica, internalizándolos y haciendo que la actividad económica sea menos rentable. Con una fuerte defensa de los derechos de propiedad, los agentes del mercado se verían libres para tratar de encontrar formas más rentables de explotar sus tierras, de modo que surgirían otras alternativas económicas menos contaminantes, una vez que las actividades más contaminantes serían más costosas. Tal como han expuesto autores como Ronald Coase o Walter Block, un ejemplo de alto grado de defensa de los derechos de propiedad es el modelo basado en la jurisprudencia anglosajona en la Inglaterra anterior a 1840-1850. En esa época, con una invasión de la propiedad privada, con contaminación, a través de polución o residuos, las compañías contaminantes tenían que pagar daños y pérdidas a los afectados. De tal manera, que no importaba cuán grande era la 
compañía, sus intenciones o su supuesto papel en el crecimiento económico, sino solamente los derechos de propiedad, es decir, si la propiedad estaba siendo invadida o no. Este escenario cambió cuando la noción de que había un interés público, que estaba por encima de los derechos de propiedad, se fortaleció (BLOCK, 1990).

El Estado, a través del bien público, impide la existencia de un mercado para la contaminación y la polución producida. Como dice Murray Rothbard, la falta de un mercado conduce a la falta de incentivos financieros para que los consumidores elijan una opción menos contaminante. Es decir, el producto que más contamina, tanto cuando se produce como durante y después del consumo, será más caro para el consumidor, una vez que cada etapa en el que el bien produce contaminación, elevará el precio del producto final para los consumidores. De esta forma, los consumidores coordinarán su comportamiento, eligiendo opciones menos contaminantes. Como los precios, estimados a priori por los empresarios, son los responsables por determinar los costes, los empresarios tendrán que reasignar sus recursos en producciones menos contaminantes para no tener pérdidas. El mercado, a través de los derechos de propiedad, es la forma más eficiente de combatir la contaminación (ROTHBARD, 1982).

Sin derechos de propiedad, no es posible determinar objetivamente los costes de las externalidades. Es decir, no se puede conocer el coste social de la contaminación. Al tratar de determinar el coste de la contaminación a través de impuestos y regulaciones, el Estado lo hace de manera arbitraria, puesto que no existen precios de mercado del bien público y no se pueden hacer comparaciones interpersonales de utilidad.

Tal y como señala Murray Rothbard, "The coerced individual loses in utility as a result of the intervention, for his action has been changed by its impact." (ROTHBARD, 1970, p. 11). A partir de este concepto, entendemos que no existe un problema real si los consumidores demandan una actividad más contaminante en mayor medida. Una vez que están dispuestos a pagar el coste adicional, por entender que obtienen un mayor beneficio social de ese producto. O como expuesto por Ludwig von Mises:

El hombre tiene que pagar un precio por todos sus logros. Las personas pagan el precio si creen que los beneficios que se derivarán de lo que se adquirirá son mayores que las desventajas que conlleva el sacrificar alguna otra cosa [...] El referirse a lo maravilloso de la naturaleza virgen es ocioso si no se tiene en cuenta lo que el hombre ha obtenido "profanando" la naturaleza. Las maravillas de la tierra eran en realidad espléndidas cuando pocos visitantes podían acceder a ellas. El turismo comercialmente organizado las ha puesto al alcance de muchos. Quien piensa que es una lástima que no pueda estar solo en una montaña, olvida que él mismo probablemente no podría haber llegado si el comercio no hubiera proporcionado todas las facilidades requeridas. [...] Dan por sentados todos sus logros, pero lo culpan de la desaparición de algunos goces que son incompatibles con él y de algunas imperfecciones que todavía empañan sus consecuciones. Se olvidan de que la humanidad ha tenido que pagar un precio por sus conquistas, precio pagado gustosamente, ya que la gente cree que el beneficio obtenido, la prolongación del promedio de vida, por ejemplo, es más deseable. (MISES, 1975, p. 194-195) 


\subsection{Visión a Corto Plazo}

Autores como Chang, Andreoni y Kuan sostienen la idea de que el estado, al tener una mayor capacidad de inversión, va a dedicarse a proyectos de inversión de más larga duración. En contraste, las empresas tendrían una visión a corto plazo para afrontar a la incertidumbre. Es decir, una alta preferencia temporal, terminan optando por la deforestación y la contaminación en lugar de la conservación del medio ambiente. Este punto de vista justificó la creación de instituciones forestales centralizadas, como garantizadoras del bien público, suponiendo que los usuarios locales del bosque estarían más preocupados por los beneficios inmediatos y privados.

En realidad, el Estado no consigue hacer frente a la incertidumbre de manera más eficiente que el empresario, puesto que no posee los incentivos ni los medios para que están a la disposición del empresario cuando este actúa en el mercado. Como nos explica Oscar Carreiro:

El Estado, al afrontar cualquier actividad económica, se encuentra sometido igualmente a la incertidumbre. La pregunta que hay que realizar es si puede afrontar la incertidumbre de mejor manera que los empresarios privados y la respuesta es que no, ya que no tiene ni los incentivos ni los medios para ello. No tiene los incentivos porque el Estado no se juega su propio dinero, ni tiene los medios porque no puede obtener la información necesaria para la toma de decisiones, ya que esta información se genera por el propio proceso de mercado y la intervención gubernamental previene su creación y difusión (CARREIRO, 2017, p. 161).

Como señala Rothbard, es justamente los propietarios privados quienes tienen una visión a largo plazo porque quieren que se mantenga el valor de su propiedad (ROTHBARD, 1970, p. 36). Por lo tanto, una empresa solo puede seguir siendo rentable con el tiempo, planeándose para la demanda presente y futura. Las empresas que explotan imprudentemente los recursos, ya sea por descuido o ignorancia, hacen que sea más rentable para los empresarios más perspicaces, comprar y mantener una gran cantidad de un determinado recurso fuera del mercado, hasta que su precio aumente como resultado de la explotación. Los agentes del mercado, a través de la anticipación, actuarán, llevando a un aumento en los precios. Este precio más alto, es una información para los agentes del mercado de que un recurso en particular es muy valioso y vale la pena preservarlo. Además, hará que los empresarios, que compran este recurso natural como factor de producción, busquen alternativas para mejorar su eficiencia o reemplazarlo

Si el precio de un recurso natural en particular, como la madera, aumenta gradualmente, se producirá gradualmente una transición a una fuente alternativa a la madera. Esto asegurará un suministro estable del recurso y reducirá la demanda de madera. Los precios indicarán a los consumidores que este es un recurso que vale la pena conservar financieramente, para que coordinen su comportamiento frente a las necesidades de los demás. Los consumidores utilizarán otros productos que realizan una función similar porque son económicamente ventajosos. 


\subsection{Leyes de Conservación y Escasez de Recursos}

Las leyes de conservación apuntan a limitar el uso de recursos naturales no renovables, forzando el uso y la producción de recursos renovables. Por lo tanto, estas leyes tienen la intención de reducir coercitivamente la preferencia temporal de la sociedad, promulgando que ciertos recursos deben ser preservados para que estén disponibles para las generaciones futuras. Las leyes suponen que las preferencias temporales de la sociedad conducirían naturalmente al agotamiento de los recursos, ya que una mayor preferencia temporal implica un mayor consumo. Sin embargo, cuando el gobierno prioriza el futuro a expensas del presente, lo hace a pesar de la sociedad, que ya ha decidido, en función de los tipos de interés, cuál será su estructura intertemporal de bienes de capital (ROTHBARD, 1970, p. 35-36). Además de eso, en algún momento, dicho futuro será el presente y dichos recursos naturales deben de madurar en forma de bienes de consumo. Como retrata Anthony Scott: "Most of our progress has taken the form of converting natural resources into more desirable forms of wealth. If man had prized natural resources above his own product, he would doubtless have remained savage, practicing 'conservatism'". (SCOTT, 1983, p. 11).

El hecho es que las leyes de conservación requerirán una mayor inversión en recursos naturales. Se utilizarán recursos alternativos para mantener los recursos renovables y se impondrá una mayor reserva de recursos agotables. Sin embargo, como los ahorros de la sociedad no han aumentado, esta inversión tendrá que trasladarse de áreas en las que en un libre mercado sería más productiva, provocando una distorsión de la estructura productiva a una que no satisface el real deseo de los consumidores. Además, Rothbard describe que la exclusión de los recursos y de la tierra del mercado ineludiblemente genera privilegios de monopolio para los propietarios remanentes, una vez que, al restringir el suministro de estos bienes, sus precios aumentan artificialmente (ROTHBARD, 1970, p. 37-38).

Los problemas relacionados con la preservación de los bosques, las proyecciones para desastres ambientales y las consecuencias de la deforestación se abordan y analizan desde un punto de vista estático, en una cosmovisión utópica donde no hay más intercambios y el empresario es un agente pasivo. Nada más lejos de la realidad. Al aplicar la perspectiva dinámica, tenemos que el empresario, sin barreras coactivas, es libre para crear soluciones para el uso sustentable de los bosques de manera innovadora. El empresario provoca cambios en el mercado al crear nueva información, cambiando los fines y los medios que persiguen los demás. Por lo tanto, cualquier proyección de desastre ambiental, hecha desde un marco de eficiencia estática, no puede realizarse. En el mercado, el futuro es un "por hacer" y no un "por venir". La gama de soluciones a los problemas que impregnan la vida de los seres humanos es ilimitada a medida que aumenta la división del conocimiento y no se impide el libre ejercicio de la función empresarial. Un ejemplo de lo que estamos hablando es la crisis del estiércol de caballo que ocurrió en ciudades como Nueva York y Londres a fines del siglo XIX. En ese momento, el caballo era indispensable en los centros urbanos para el transporte de personas y mercancías. Sin embargo, la cantidad de estiércol producido fue tan grande, que se hizo una proyección de que en 50 años Londres estaría bajo 9 pies de estiércol. Las autoridades celebraron reuniones en un intento por resolver el problema aparente. Lo que no contaron, 
sin embargo, fue que el problema desaparecería en unos pocos años, debido a la creación, por parte de empresarios que buscaban beneficios, de los primeros coches (DAVIES, 2004).

El ser humano, con su creatividad empresarial, se reinventa. Por lo tanto, la relativa escasez de recursos de los desastres naturales, del cambio climático y de la contaminación, no son un obstáculo para que las personas resuelvan estos problemas, creando formas previamente impensables para enfrentar la adversidad y continuar mejorando su nivel de vida. No es de extrañar que los recursos que tenemos a nuestra disposición hoy en día se encuentren en la naturaleza en cantidades más pequeñas que en el pasado. Sin embargo, la acumulación de capital y la creación de nuevas tecnologías nos han permitido aumentar la productividad para que tengamos más y más bienes de consumo a un precio cada vez más accesible. Un proceso que hace rentable el uso de recursos alternativos, de modo que un recurso que hoy se utiliza puede no ser muy demandado en el futuro". O como dice Rothbard, "Artificial conservation will wastefully prolong resources beyond the time when they have become obsolete" (ROTHBARD, 1970, p. 36).

\section{Mercado Ecológico}

Los bienes ambientales en general y las oportunidades de recreación forestal en particular tienen una demanda elástica. Es decir, a medida que aumenta el ingreso de las personas, también lo hace la demanda por dichos bienes. Por lo tanto, un aumento en la posibilidad de pagar por más actividades al aire libre significa que hay una mayor posibilidad de obtener ganancias de estas actividades y de productos relacionados con ella.

Since World War II, incomes for United States citizens have been rising dramatically, increasing the willingness of Americans to pay more for outdoor opportunities. Total visits to Yellowstone National Park in 1997 were 32 percent higher than in 1986; during the same period,entrance fees rose from $\$ 2$ to $\$ 20$ per vehicle. Comparing figures from 1960 and 1996, average expenditures per individual,in real terms,more than doubled for fishing and nearly quadrupled for hunting. As a result,a growing number of farmers and ranchers in the United States have found that hunters are willing to pay for the opportunity to hunt on their land. (ANDERSEN; LEAL, 2001, p. 59).

Es importante comprender que el capital tiende a ir al sector productivo en el que la sociedad lo demanda más. Por lo tanto, el empresario empleará capital en un tipo particular de producción hasta que el precio de un producto determinado sea igual a su coste marginal. Un agricultor que quema parte de su tierra para producir soja verá que el precio de la soja baja a medida que produce más, y el coste de la producción de soja aumentará a medida que demandará más insumos para esta actividad. Mientras que, teniendo el libre uso de su propiedad, verá que el beneficio que obtiene al preservar el bosque será mayor que el agrícola.

\footnotetext{
${ }^{2}$ Daniel Jay Brown (1804-1867) quien, en la obra de 1832 The Sylva Americana, preocupado por el consumo de madera, cuestionó: "Whence shall we procure supplies of timber fifty years hence for the continuance of our navy?". (SCOTT, 1983, p. 37)
} 
Cuando esto suceda, el mercado transferirá capital para la preservación y explotación sostenible de los bosques.

Es el sector privado el que debe ofrecer el bosque para uso recreativo. Cuando el gobierno decide ofrecer este servicio a la población, su naturaleza política y el propósito de ganar votos, hacen que ofrezca el bien gratis o a un precio muy bajo. Esto no solo genera servicios de baja calidad y uso excesivo, sino que tiene consecuencias aún más graves. El bien público distorsiona el precio de mercado de este tipo de actividad comercial, por lo que se convierte en una actividad no rentable. Por lo tanto, los productores no desviarán recursos de actividades productivas alternativas, como la agricultura o la ganadería, al uso recreativo, ya que el precio de estas actividades no es lo suficientemente alto para generar retornos positivos.

Sin embargo, la provisión de este tipo de actividad está relacionada con cuánto los consumidores están dispuestos a pagar por ella. Si los consumidores no valoran o no están dispuestos a pagar demasiado, no será posible explotarla económicamente para este propósito. Por lo tanto, es importante que el propietario sea libre de explotar la tierra y generar el valor necesario para hacer posible el uso sostenible.

Los pueblos indígenas son un ejemplo de lo que estamos hablando. Al no tener un control total sobre el uso de la propiedad, como lo regula FUNAI, no pueden encontrar la mejor manera de explotarlo económicamente. Los indios controlan la entrada a sus tierras, pero el Estado limita la forma en que se debe usar la tierra. Este tipo de ley impide la provisión de actividades relacionadas con la vida silvestre. Una solución sería el establecimiento de lotes individuales de propiedad, o una organización local y descentralizada de la gestión de los recursos comunes (RUC) en la línea de Ostrom. Sin embargo, hace falta que el Estado no intervenga y que haya exclusión de los recursos.

El modelo de arrendamiento tampoco es una solución. Según Rothbard, cuando el Estado arrienda tierras a tribus indígenas, ganaderas o madereras, no hay incentivos para preservar el recurso. El hecho de que no sean propietarios les hará usar los recursos para obtener mayores beneficios en el presente, cayendo en el problema de la tragedia de los bienes comunes. Por otro lado, cuando existen derechos de propiedad sobre la tierra, existen incentivos para preservar y maximizar el valor presente de cada recurso, ya que el agotamiento del recurso reduciría el valor de su propiedad (Figura 1). Para preservar su capital, el propietario explotará el recurso a una tasa que tenderá a ser igual a la tasa social de preferencia temporal (ROTHBARD, 1970, p. 37).

The same misguided view of markets for wildlife led to the abolishment of the legal ivory market by the 1989 CITES treaty. Western conservation and animal rights groups that pushed for the ban interpreted the legal market for ivory as the culprit in the decline of African elephants due to poaching. While the ban was successful in stopping the legal trade in ivory, which resulted in lost revenues for local conservation efforts in African countries, there is little evidence that it stopped poaching. Poaching, in fact, has "continued without interruption in Africa's tropical forests." In Zambia, Tanzania, Cameroon, Ivory Coast, and Zaire, poaching has slowed, but that has been due to increased law enforcement financed, in part, by outside donations. The Kenyan government does attribute a 32 percent increase in its elephants between 1989 and 1991 to the ban, but it is more likely due to elephant 
immigration from adjacent countries or inaccurate population estimates. In Zimbabwe and Botswana,elephant populations have thrived for years because of successful conservation programs that capitalized on the legal ivory trade. In those countries, local people have a strong economic interest in protecting elephants from poaching because the revenue from tusks and hides and a portion of the money made from selling hunting permits go to local communities where the elephants live. It is property rights and markets that account for the rapid elephant population growth in Zimbabwe and Botswana and for the declining population in the rest of Africa. (ANDERSEN; LEAL, 2001, p. 64-65)

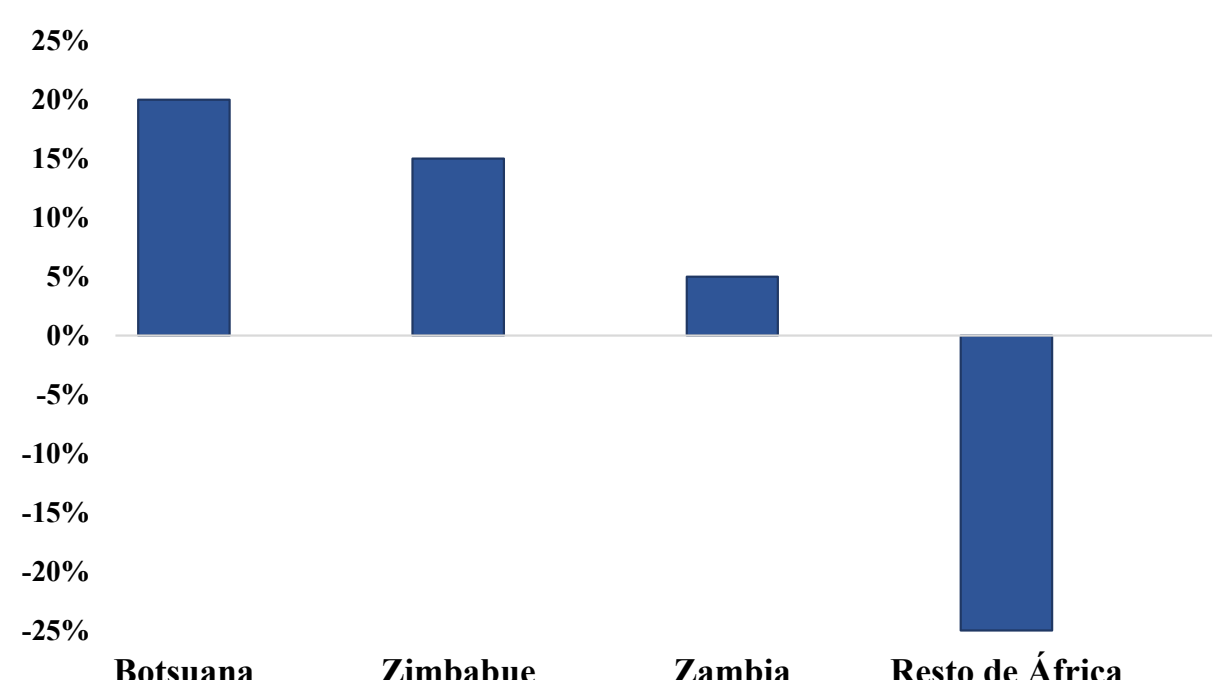

Figura 1. Cambio de la población de elefantes entre los años 1989-1995.

Fonte: Andersen e Leal (2001, p. 65).

Las regulaciones actúan como un desincentivo para la creación de actividades que protejan el medio ambiente y la vida silvestre. Como lo expresó un terrateniente estadounidense: "Look, you don't understand. We would like to do our share for wildlife but we are afraid if we create something worthwhile the public will want what we have. It's just plain easier and a lot safer to sterilize the land". (ANDERSON; LEAL, 2001, p. 72).

Los subsidios gubernamentales a las actividades agrícolas también distorsionan los precios del mercado. Al reducir los costes involucrados en estas actividades, las hacen mucho más rentables que otras actividades económicas que los consumidores demandan más. Esto hace que se utilice tierra de baja productividad agrícola para la agricultura, cuando su uso más productivo sería recreativo, por ejemplo.

Por lo tanto, los derechos de propiedad no solo deben garantizarse, sino que el Estado no debe interferir con el funcionamiento del mercado. Al hacerlo, distorsiona los precios, hace que el capital fluya hacia áreas menos productivas y, en última instancia, hace que se evite lo que se buscaba con la intervención estatal. Es decir, menor productividad agrícola y mayor deforestación. 


\section{Amazonia}

Brasil tiene un área de 8.4 millones de kilómetros cuadrados, de los cuales 5 millones (60 por ciento) corresponden a la Amazonía Legal. Su población, sin embargo, corresponde al 12 por ciento de un total de 209 millones de habitantes de Brasil. El Amazonas es la selva tropical más grande del mundo (HEUSER, 2018, p. 8).

Dado el tamaño y la importancia global de la Amazonía, las intenciones de los acuerdos internacionales de protección ambiental terminan enfocándose en Brasil. En consecuencia, los movimientos ambientales de 1980 culminaron en la conferencia Río 93, donde se acordó entre los países del G7, ayudar financieramente y proteger la selva amazónica. Posteriormente, Brasil firmó varios acuerdos internacionales de protección ambiental y sobre el cambio climático, que culminaron en el acuerdo de París (HEUSER, 2018, p. 11).

Desde Río 93, el Estado brasileño y los donantes internacionales han implementado varios proyectos para proteger la selva amazónica. De los más relevantes, podemos destacar el Proyecto de Demarcación de Tierras Indígenas y el Programa de Áreas Protegidas del Amazonas (ARPA). El país se ha fijado el objetivo de proteger los recursos naturales en las tierras indígenas, que son tema de conflictos locales, una vez que se puede obtener usos más productivos para las áreas con su estado legal actual.

\subsection{Reservas Indígenas}

Las reservas indígenas en Brasil, que comprenden el 20 por ciento de la Amazonía legal, son de vital importancia para comprender los problemas de derechos de propiedad en la Amazonía. Según la ONU, "Indigenous peoples have the right to the lands, territories and resources which they have traditionally owned, occupied or otherwise used or acquired" (UNITED NATIONS, 2008, s/p). Sin embargo, me gustaría aplicar el concepto Homestead porque creo que es la mejor manera, desde un punto de vista de eficiencia dinámica, para definir los derechos de propiedad. La demarcación de tierras indígenas ha sido utilizada como una de las principales formas de protección ambiental por la agenda de la ONU. Sin embargo, los pueblos indígenas, que corresponden al 0,4\% de la población brasileña, no tienen un derecho de propiedad real sobre las tierras de la selva amazónica (HEUSER, 2018, p. 30).

Las reservas indígenas son una forma de área protegida, como un santuario de animales. Sólo que, en ese caso, con personas. A los indios se les permite usar los árboles y los recursos naturales sobre el suelo en una cantidad que satisface solamente sus necesidades básicas. No se les permite ningún acuerdo comercial, y mucho menos el intercambio de bienes. Las reservas indígenas pertenecen al Estado brasileño, que es responsable por proteger el territorio de intrusos (HEUSER, 2018, p. 59).

Esta situación legal que se les presenta no permite que los indios puedan desarrollar comercialmente el área. Por lo tanto, una región con un gran potencial para la explotación económica, y al mismo tiempo con una población muy pobre, termina siendo blanco de conflictos entre los indios y sus vecinos, así como entre los agricultores, los madereros y las 
empresas de extracción de minerales. Como la tierra es propiedad del Estado, el control sobre la propiedad es muy ineficiente, una vez que no hay un propietario con incentivos financieros para proteger su tierra y crear las soluciones adecuadas para eso. Del mismo modo, al no garantizar los derechos de propiedad, la tierra se convierte en el blanco de todos los que la ven como una forma de explotación económica, cayendo en un caso típico de la tragedia de los bienes comunes, y donde formas alternativas de explotación, que protegerían el bosque, se vuelven imposibles por las regulaciones y la falta de derechos de propiedad.

$\mathrm{Al}$ no poder utilizar sus tierras de la manera que más les convenga, la calidad de vida entre los pueblos indígenas es muy baja. Sus ingresos provienen principalmente del turismo, que actúa como una especie de safari, y de programas del gobierno de redistribución de renta. En algunos casos, cuando una autopista pasa por la reserva india, se cobra un peaje. Esta rara oportunidad de aumentar los ingresos ha llevado a los pueblos indígenas a establecerse cerca de las carreteras (HEUSER, 2018, p. 60). Como resultado, el acceso a la salud es muy bajo o inexistente en las reservas indígenas, por lo que la tasa de mortalidad infantil es mucho más alta que en el resto del país. El indio no puede ser tratado como un animal en peligro de extinción, que debe ser preservado y protegido, encerrado dentro de su hábitat natural, la selva.

As result of these flaws, the social situation is in general very difficult inside the Indigenous territories of the Amazon. Health and the social indices are among the wort in Brazil: infant mortality rate is much higher than the national, tuberculosis or malaria prevalence are extremely high $(34,35)$, nutrition problems are commons and alcoholism, or suicide are widespread. Indigenous people must always keep a political pressure on the federal government (for instance by invading regional offices of the health administration) in order to granted the necessary. (LE TOURNEAU, 2015)

Como si eso fuera poco, la tasa de deforestación en las reservas indígenas tampoco justifica un programa de demarcación con el propósito de proteger el medio ambiente (HEUSER, 2018, p. 84).

\subsection{Otras Políticas}

Además de la demarcación de las reservas indígenas, las otras políticas de preservación ambiental utilizadas por el gobierno brasileño consisten en: 1) Protección estricta de ciertas áreas, a través de regulaciones que les otorgan el estatus de Área Protegida. Las áreas protegidas son manejadas por patrulleros que hacen cumplir y aseguran las fronteras. 2) Áreas de uso sostenible, donde se permite el uso sostenible de los recursos. En este caso, se supone que el gobierno federal y local protegerá el área y detendrá a los intrusos. 3) Política de refuerzo de la ley, que faculta al IBAMA (Instituto Brasileño de Medio Ambiente y Recursos Naturales Renovables) para monitorear la deforestación a través de imágenes satelitales. A través de las imágenes y su presupuesto, el IBAMA interviene al detectar alguna actividad de deforestación, confiscando el equipo y abriendo un enjuiciamiento legal. Por último, el gobierno federal evalúa la efectividad de los municipios para reducir la deforestación y puede enmarcarlos en incumplimiento del código forestal brasileño, lo que dificulta la transferencia de fondos (HEUSER, 2018, p. 127-128). 
Como hemos visto, estas medidas no están siendo satisfactorias para reducir la deforestación en la selva amazónica. Se observan muchos focos de incendios todos los años, así como constantes conflictos entre diferentes grupos de interés en la región. El Estado no tiene otra salida que decir que el problema se debe a la falta de recursos para combatir la deforestación. De esta manera, los recursos cada vez más escasos, se desvían para el logro de un fin de modo ineficiente. Incluso si las políticas gubernamentales logren presentar alguna mejora en la preservación de los bosques, sigue existiendo el problema del cálculo económico. Es decir, no se puede saber la manera más barata para lograr los resultados que se pretende, de modo que haya el despilfarro y la mala asignación de recursos escasos que son más demandados, por los seres humanos, en otros sitios, para atender a sus necesidades más urgentes.

El "Fundo Amazônia" recibe \$ 380 millones de dólares por año. Este recurso se envía al gobierno brasileño a través del programa internacional REDD+ (FUNDO AMAZÔNIA, 2019). Se puede argumentar mucho sobre la voluntad o la falta de voluntad de un gobierno en particular para hacer de este recurso un medio para proteger el bosque. La verdad es que esta cantidad nunca será suficiente y los países deberían dejar de donar. No es que no tenga ninguna utilidad, la donación tiene un efecto de agujero en la pared, pero no resuelve el problema. Hay varias razones por las cuales los programas de donación como REDD + no son efectivos para frenar la deforestación. Primero, los agricultores que tienen los costes más bajos de reducir la deforestación son los que tienen más probabilidades de ingresar a este proyecto (PERSSON; ALPIZAR, 2013). Como resultado, el proyecto puede terminar pagando a algunos agricultores por no hacer nada diferente de lo que hubieran hecho en ausencia de cualquier pago. El impacto del proyecto termina siendo muy pequeño, a pesar del alto coste involucrado. En segundo lugar, el impacto del proyecto, si lo hay, puede compensarse con el hecho de que el proyecto aumenta la deforestación entre los no participantes. Si la población da una mayor utilidad marginal a la agricultura, ella sólo podrá ser superada con la existencia de mercados alternativos que puedan competir con la actividad agrícola. En tercero, "In the most extreme cases, for example, if some landowners use all payments to buy chainsaws to clear more forest for cattle pasture, negative spillovers could exceed the positive impacts of the project" (WUNDER, 2007).

Una cosa que se debe tenerse en cuenta, es que la mayoría de las áreas de protección ambiental en la selva amazónica, están demarcadas donde hay una población local muy pobre (IBGE). Por lo tanto, mantener la región como un área de explotación económica limitada juega un papel importante en el mantenimiento de la pobreza local. No solo eso, el hecho de que sea pobre significa que la población local no puede pagar los costes de preservación de la región. Como resultado, los incentivos para la explotación local son muy grandes y se realizarán donde sea posible obtener algún retorno financiero que complemente el ingreso de subsistencia, aunque que sea ilegal. Por lo tanto, tenemos un escenario lleno de incentivos para la deforestación y la explotación local de cualquier actividad que no conserve el bosque, ya sea agricultura, ganadería, extracción de madera, etc. Cualquier actividad que pueda generar beneficios para la población local al paso que protege el bosque y su biodiversidad, y así competir con otras actividades que deforestan y contaminan el bosque, se ven imposibilitadas por barreras estatales que obstaculizan y evitan la formación de un mercado forestal. 
Según Jeffrey Sayer, las personas empezaron a valorar los paisajes naturales, la vida silvestre, las plantas raras y la naturaleza en general, a partir del momento en que pudieran satisfacer sus necesidades más básicas y hubiera tiempo para dedicarse a ello, de manera que la naturaleza pudiera ser vista como algo a preservar (SAYER et al., 2004). Sin embargo, esto sólo es posible debido al aumento de la riqueza y de la productividad del factor trabajo. Por lo tanto, alguien con una buena posición financiera tiende a dar una mayor utilidad marginal a los problemas ambientales que a una cierta cantidad de dinero, mientras que una persona pobre tenderá a dar una mayor utilidad marginal a la misma cantidad de dinero que a la naturaleza.

Impedir que las personas exploten los recursos naturales es poner trabas al crecimiento económico. Esto, dificulta que las personas con un bajo nivel de vida aumenten su renta hasta el punto en que se puedan satisfacer sus necesidades más básicas. Es por ese motivo que las preocupaciones ambientales a menudo provienen de los países más ricos (ENVIROMENTAK PERFORMANCE INDEX, 2018) (Figura 2), donde la utilidad marginal de la naturaleza tiende a ser más alta y se puede pagar el coste de formas de producción menos contaminantes. Ali no ser posible hacer comparaciones interpersonales de utilidad y adoptando nuestro criterio dinámico de eficiencia, deducimos que el respecto a la propiedad privada mueve hacia la derecha la curva de posibilidades máximas de producción, permitiendo el desarrollo de tecnologías menos contaminantes y el enriquecimiento de la población.

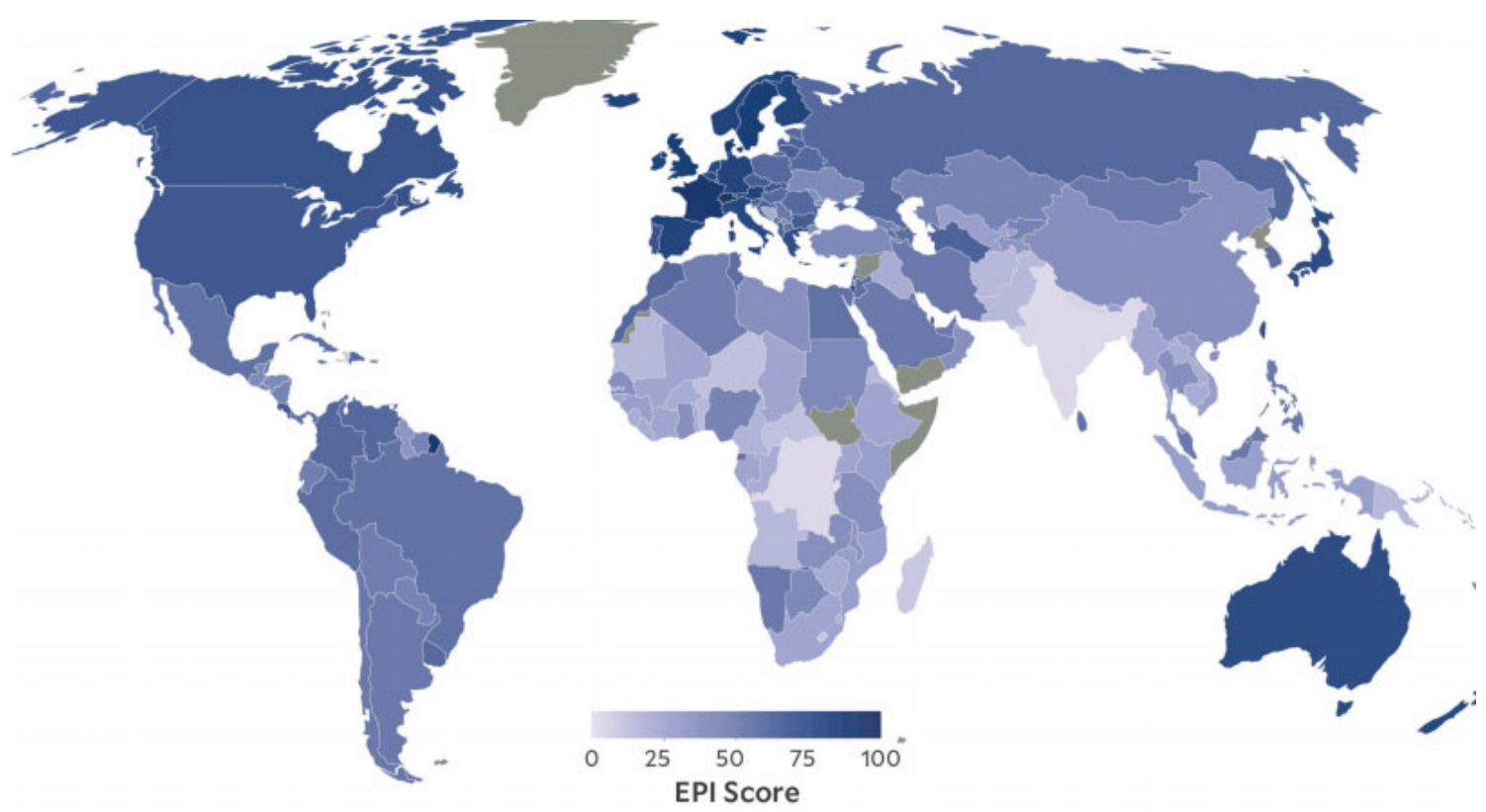

Figura 2. Índice de desempeño ambiental.

Fonte: Enviromental Performance Index (2018). 


\section{Conclusión}

Concluimos que la Amazonía debe ser privatizada. La propiedad estatal del bosque genera un problema de externalidades negativas, como se ve en la tragedia de los comunes. La única forma racional de internalizar los costes de la deforestación y de la contaminación es a través de los derechos de propiedad privada sobre los bosques. Por lo tanto, los derechos de propiedad deben garantizarse con toda la fuerza de la ley, ya que son la condición sine qua non para el surgimiento del mercado. Sólo de esta forma se podrá lograr un crecimiento económico que sea verdaderamente sostenible, una vez que el ser humano, al poder aprovecharse del resultado de su genuino acto empresarial, tendrá menos barreras para el ejercicio de la función empresarial. Se debe reconocer el papel del empresario como agente creador y coordinador de los desajustes sociales que, a través de un proceso dinámico competitivo, es capaz de descubrir las mejores formas de hacer frente a los problemas reales de la sociedad.

La salida de la Amazonía de las manos del Estado, migrando hacia sector privado, permitirá la aparición de precios reales de mercado de los factores de producción, teniendo una asignación racional de recursos, resolviendo el problema del cálculo económico. Además, permitirá la creación de nuevos mercados, muchos de los cuales serán ecológicos y sostenibles, lo que mejorará la conservación de la región y permitirá explotar el gran potencial económico del bosque.

La privatización, además de aumentar la eficiencia en la utilización de los recursos y reducir el despilfarro, establece un marco legal para que haya eficiencia dinámica, aumentando las posibilidades máximas de producción.

El indio debe de tener derechos sobre su propiedad y poder explotarla e intercambiarla si así lo desea. Esto no sólo le permitirá elevar su nivel de vida, sino que también es más justo, una vez que es el primero en mezclar su trabajo con la tierra.

Entendemos que el coste social de proteger el medio ambiente debe ser un coste que la sociedad está dispuesta a pagar. Siendo el mercado el mediador por excelencia de la acción de cada uno de los individuos.

La privatización es la única forma de proteger la Amazonía y su biodiversidad. Probablemente, la extensión de la Amazonia se reduciría un poco, pero la enorme parte no deforestada estaría mucho mejor protegida.

\section{Referencias}

ANDERSON, T.; LEAL, D. Free Market Environmentalism. New York: Palgrave, 2001.

BLOCK, W. Economics and the Environment: Environmental Problems, Private Property Rights Solutions. Canada: The Fraser Institute, 1990.

CARREIRO, O. R. Las Políticas de la Industrialización. Santiago de Compostela: Universidad de Santiago de Compostela, 2017. 
DAVIES, S. Foundation for Economic Education, 2004. Disponible: https://fee.org/articles/the-great-horsemanure-crisis-of-1894/. Acceso en: 30 nov 2019.

ENVIROMENTAL PERFORMANCE INDEX. Global metrics for the environment: Ranking countryperformance on high-priority environmental issues. 2018. Disponible en: https://epi.envirocenter.yale.edu/downloads/ epi2018policymakerssummaryv01.pdf. Acceso en: 1 dic 2019.

FUNAI. Fundação Nacional do Índio. Ministério da Justiça e Segurança Pública, s/f. Disponible en: http://www. funai.gov.br/index.php/quem-somos. Acceso em: 02 dic 2019.

FUNDO AMAZÔNIA. Junio de 2019. FUNDO AMAZÔNIA. Disponible en: http://www.fundoamazonia.gov. br/export/sites/default/pt/galleries/documentos/rafa/RAFA_2018_port.pdf. Acceso en: 2 dic 2019.

HARDIN, G. Tragedy of the Commons. Floresce: Science, 1968.

HEUSER, S. The Effectiveness of Enviromental Policies on Reducing Deforestation in tha Brazilian Amazon. Maastricht: Boekenplan, 2018.

HOPPE, H.-H. A Theory of Socialism and Capitalism: economics, politics, and ethics. Las Vegas: University of Nevada, 1989.

HUERTA DE SOTO, J. La Teoría de la Eficiencia Dinámica. Procesos de Mercado: Revista Europea de Economía Política, v.1, n.1, 2004.

HUETA DE SOTO, J. Socialismo, Cálculo Económico y Función Empresarial. Madrid: Unión Editorial, 2005.

IBGE. Instituto Brasileiro de Geografia e Estatística. IBGE. Informações Ambientais. Disponible en: https:// www.ibge.gov.br/geociencias/informacoes-ambientais/geomorfologia/15819-amazonia-legal.html?=\&t=o-que-e. Acceso en: 31 nov 2019.

KIRZNER, I. Competition and Emtrepreneurship. Chicago: University of Chicago Press, 1973.

LE TOURNEAU, F. M. The Sustainability Challenges of Indigenous territories. Amsterdã: Elsevier, 2015.

LOCKE, J. The Two Treatises of Government. London: A. Millar, 1689.

MISES, L. v. Economic Calculation in the Socialist Commonwealth. Auburn: Mises Institute, 1920.

MISES, L. v. Teoría e Historia. Madrid: Unión Editorial, 1975.

MISES, L. v. La Acción Humana: Tratado de Economía. Madrid: Unión Editorial, 2011.

OSTROM, E. El Gobierno de los Bienes Comunes. La Evolución de las Instituciones de Acción Colectiva. Ciudad de México: Universidad Nacional Autónoma de México, 2000.

PERSSON, U.; ALPÍZAR, F. Conditional Cash Transfers and Payments for Environmental Services - A Conceptual Framework for Explaining and Judging Differences in Outcomes. World Development, v.43, p. 124-137, 2013.

ROTHBARD, M. Power \& Market: Government and the Economy. Kansas City: Sheed Andrews and Mcmeel, Inc., 1970.

ROTHBARD, M. Law, Property Rights, and Air Polution. Cato Journal, v.2, n.01, p. 55-99, 1982.

SAYER, J. et al. The Implications for Biodiversity Conservation of Decentralised Forest Resources Management. IUCN and WWF, 2004.

SCOTT, A. Natural Resources: The Economics of Conservation. Ottawa: Carleton University Press, 1983. 
UNITED NATIONS. United Nations Declaration. 2008. Disponible en: https://www.un.org/esa/socdev/unpfii/ documents/DRIPS_en.pdf. Acceso en: 1 dic 2019.

WUNDER, S. The efficiency of payments for environmental services in tropical conservation. Conservation biology, 2007.

RECIVED: $01 / 01 / 2020$

APPROVED: 01/04/2020 